\title{
Influencia de las tecnologías de la información y comunicación en el desarrollo investigativo escolar ${ }^{1}$
}

\section{Influence of information and communication technologies on school research development}

\author{
DOI: http://dx.doi.org/10.17981/cultedusoc.9.3.2018.107
}

Artículo de investigación. Fecha de recepción: 15/06/2018. Fecha de aceptación: 27/11/2018

\author{
Heidy Granados-Viloria ${ }^{2}$ \\ Yolmaris Gutierrez-Guerrero; Arlem Manotas-Palacios y Yemmys Ruiz-Martinez \\ Institución Educativa Departamental Rural de Palmira (Colombia) \\ heidygranadoviloria@hotmail.com
}

Para citar este artículo:

Granados-Viloria, H., Gutierrez-Guerrero, Y., Manotas-Palacios, A. y Ruiz-Martinez, Y. (2018). Influencia de las tecnologías de la información y comunicación en el desarrollo investigativo escolar. Cultura. Educación y Sociedad 9(3), 905-912. DOI: http://dx.doi. org/10.17981/cultedusoc.9.3.2018.107

\section{Resumen}

Es considerable la importancia de las tecnologías en la búsqueda de soluciones a las diversas problemáticas de la sociedad, entre ellas las educativas y las de investigación. Por lo anterior se realizó la investigación, cuyo objetivo fue analizar la importancia de las tecnologías de la información y comunicación en el desarrollo investigativo escolar. Metodológicamente se guió bajo el enfoque cualitativo utilizando un diseño de investigación acción participación, la unidad de análisis estuvo conformada por diez (10) estudiantes de sexto (6) de secundaria de la Institución Educativa Departamental rural de Palmira, se utilizaron técnicas como la observación participante y la entrevista en profundidad. Los resultados evidenciaron que las influencias de las tecnologías en las aulas de clase impactan de manera positiva, aumentando el interés investigativo de los estudiantes, propiciando a la utilización de herramientas innovadoras y necesarias para el desarrollo de la clase.

Palabras clave: Tecnología, desarrollo investigativo, estudiantes, información.

\section{Abstract}

The effort that is made to give solutions to the recent technological demands of the current society is considerable. Starting from the use of computerized information and communication in school development, where they are identified, which can influence the construction of learning-teaching. Therefore, the study aimed to review the factors that influence information and communication technologies in school research development. Methodologically guided by the qualitative approach with a descriptive scope, the analysis unit was made up of ten (10) sixth (6) high school students from the Rural Departmental Educational Institution of Palmira, using techniques such as participant observation and the diary of field The results showed that the influences of the technologies in the classrooms have a positive impact, increasing the research interest of the students, favoring the use of innovative tools necessary for the development of the class.

Keywords: Technology, research development, students, information.

\footnotetext{
1 Este artículo ha sido derivado del Programa de Fortalecimiento de la Cultura Ciudadana y Democrática CT+I a través de la IEP apoyada en TIC en el Departamento de Magdalena: CICLON
}

2 Líder del grupo de investigación "Pedagogas Tecnológicas".

3 Docentes de Institución Educativa Departamental Rural de Palmira y del grupo de investigación "Pedagogas Tecnológicas".

- The author; licensee Universidad de la Costa - CUC.

Cultura, Educación y Sociedad vol. 9 no. 3, pp. 905-912. Diciembre, 2018

Barranquilla. ISSN 2389-7724 Online 


\section{Introducción}

Para el desarrollo de la presente investigación, es importante en primera instancia, identificar los principales roles y usos de las tecnologías de la información y comunicación (TIC) en la vida cotidiana, los usos evidenciados en la educación y sus princiaples tendencias en la educación escolar.

Según Waldegg (2002), las TIC son un conjunto de tecnologías desarrolladas en el ámbito de la informática y telecomunicaciones, que se representan mediante códigos de texto, imagen, sonidos, videos, entre otros; y funcionan de manera interconectada para generar nuevas posibilidades de comunicación.

Las principales características de las TIC se relacionan con:

- Emplea la infromación como materia prima para su funcionamiento.

- Puede penetrar de forma ágil, prática y rápida en todos los espacios sociales.

- Es flexible, al emplear de manera simultánea todos los códigos de información que posee, y al interconectarse en red a los sistemas tecnológicos.

- Es capaz de organizarse y reconfigurarse de manera rápida y fluida.

- Posibilita la integración de tecnologías específicas en sistemas más generales

La tecnología hoy en día hace parte de la cotidianidad de los seres humanos. Los niños nacidos en la era tecnológica se encuentran inmersos en este mundo de telecomunicaciones, internet, redes sociales y aparatos electrónicos que han suplantado los juegos, juguetes, amigos y hasta la familia en los casos más graves, esto para muchos casos, pero cabe destacar que la tecnología no se creó para separar familias ni para crear conflictos, al contrario permite ampliar en cierta medida las técnicas tecno comunicativas, a través de la creación de nuevos métodos para la adquisición del conocimiento.

En las cuales las nuevas estrategias que las escuelas están implementando son cuestionadas por los estudiantes propensos a dejarse persuadir del mal uso de la cultura digital (Barbero, 2009). A nivel público, las TIC permiten mediante el uso de la Internet, conectar a los usuarios con toda la información emitida por los diferentes nodos de red, desde cualquier lugar del mundo y en tiempo real; de esta manera, accede a casi todo lo que desee.

En los procesos de enseñanza-aprendizaje, el uso de las TIC ha conducido a nuevos cambios estructurales en las escuelas, transformando los métodos de enseñanza tradicionales a posibilidades inetractivas, didácticas y con alto contenido educativo. En este sentido, es posible emplear hoy herramientas como páginas y aplicaciones web, aulas virtuales y un sinnúmero de herramientas que dinamizan y motivan a los estudiantes y permiten que los procesos se instauren de manera más efectiva (Guerra, 2013).

En Colombia, han sido muchas las acciones realizadas en las escuelas con el uso de las TIC, las cuales han permitido generar una visión distinta del sistema educativo en un mediano y largo plazo, en el que sea posible, teniendo en cuenta los beneficios en términos de desarrollo, la incorporación de las TIC a los sectores educativos del país. En este sentido, es posible determinar la necesidad de generar espacios en los que tanto los docentes como los estudiantes puedan potencializar y enriquecer procesos pedagógicos en los que se encuentran involucrados y de esta manera sea posible el aprovechamiento al máximo de los beneficios de las TIC para los procesos de formación de los 
estudiantes, en todos los niveles académicos (Said, Irirarte, Valencia, Borjas, Ordoñez, Arellano, Román, Mestre, Payares, Lara, Jabba, Brändie, González y Mejía, 2015).

Resulta fundamental, en este sentido, que tanto los maestros como los estudiantes conozcan y se actualicen de las herramientas de las TIC en los procesos de enseñanza-aprendizaje, con el fin de ofrecer competitividad en el sector educativo y en los procesos de interacción, se generen espacios más efectivos para la adquisición de conocimientos (Cárdenas, Zemeño, Tijerina, 2013).

En materia de investigación, y de acuerdo a los postulados de Mejía y Manjarrez, (2011), la IEP (investigación como estrategia pedagógica), es una metodología encaminada a fomentar el interés en la población estudiantil por la investigación científica y tecnológica, donde el maestro acompaña a sus educandos en la búsqueda del saber a través de una acción dialógica que incluye el texto, la disciplina y el método científico e incluye el uso de las TIC para fortalecer y dinamizar los procesos.

Esta herramienta permite llevar la investigación a un contexto educativo, que además ayuda a la elaboración y creación de espacios, y de mecanismos que las han compuesto.

En ese sentido, considerar la investigación como estrategia pedagógica implica recrear un espacio de inclusión, donde se respete y se viva la diversidad haciendo evidentes los canales de la creatividad del docente, para convertir las preguntas de los grupos de aprendizaje colaborativo conformados por niños, niñas y adolescentes en una investigación, llevándolos a tomar un rol activo en el proceso de aprendizaje mediante la construcción de su propia conocimiento, potenciando así, las habilidades y destrezas de los estu- diantes, convirtiendo la educación en un vehículo de capacitación, de oportunidades y de recreación (Camargo, 2015).

Además, la investigación como estrategia pedagógica, busca lograr que asesores, maestros y maestras, niños, niñas y jóvenes, establezcan un dialogo de saberes mediado por una formación en investigación, que integrada al aula de clase permita cultivar el espíritu científico en los estudiantes desde edades tempranas, creando una cultura de investigación, que a su vez posibilite una educación basada en ciencia tecnología e innovación, convirtiéndose en un aporte para el desarrollo del país (Santacoloma, 2014).

De esta manera Herrera, (2012); sostiene que los nuevos medios de información y comunicación modifican de manera radical el rol del docente generando un contexto idóneo para el desarrollo de ciertas competencias tales como el pensamiento crítico, la autonomía, la iniciativa, el trabajo colaborativo y la responsabilidad individual.

Por lo tanto, se espera que el maestro de hoy contemple cambios en su práctica, que se actualice constantemente y envuelva a sus estudiantes en entornos de crecimiento y construcción de saberes. Por otro lado, Avendaño, Cortes y Guerrero, (2015) expresan que el uso de las tecnologías de la información y la comunicación (TIC) tienen un papel importante en el desarrollo de competencias educativas y sociales, como factores predictores del desempeño académico en estudiantes de básica primaria.

Así mismo, las TIC son tecnologías para almacenar, recuperar, procesar y comunicar la información. Existe una variedad de recursos electrónicos que se encuadran dentro del concepto de TIC como; televisión, teléfonos, videos, ordenadores, entre otros. Sin embargo, los medios más 
representativos de la sociedad actual son los ordenadores que nos permiten utilizar diferentes aplicaciones informáticas (presentaciones, aplicaciones multimedia, programas ofimáticos y redes de comunicación (Belloch, 2012).

De esta manera, las Tecnologías de la Información y las Comunicación (TIC) forman parte de la cultura tecnológica. Estas permiten ampliar las capacidades físicas y mentales de los individuos. Generando diversas posibilidades de desarrollo social y educativo. Las TIC no solo se encuentran asociadas a la informática o tecnología, sino que también incluye los medios de comunicación. En ese sentido, las TIC se convierten en un instrumento cada vez más indispensable en las instituciones educativas donde pueden realizar múltiples funcionalidades (Graells, 2012).

En la actualidad el manejo de la tecnología de una forma eficaz, es una habilidad necesaria para desenvolverse en la sociedad, este cambio de paradigma social trae consigo el cambio en el paradigma educativo. Por lo cual, la educación realizar inversiones económicas y políticas, donde su papel se fundamente en la capacidad de producir sus propias herramientas tecnológicas. Y la actualización del cuerpo docente para que se encuentre en la capacidad de responder al desafío de la actualización tecnológica. (Riveros y Mendoza, 2004).

\section{Metodología}

Se realizó un estudio de tipo cualitativo, utilizando el modelo de investigación acción- participación. La investigación cualitativa busca conocer e interpretar la realidad de los participantes a través de sus propias experiencias, entregando una información subjetiva del fenómeno de estudio. (Hernández, Fernández, y Baptista, 2010). El modelo de investi- gación acción se basa en la inclusión del investigador en donde la participación de la unidad de análisis implicada ayude a transformar la realidad a través conocer que estrategias pueda utilizar e implementarlas, en donde los colaboradores lleguen dar respuesta utilizando los recursos con los que cuentan. Con el objetivo de implementar y observar el uso de la tecnología en los estudiantes, y a su vez relacionarlo con el rendimiento académico, para así establecer la gran importancia que tiene el uso de las tecnologías durante el proceso de aprendizaje.

\section{Participantes}

En cuanto a la a la unidad de análisis que se tomó para la realización del estudio, se tomó una muestra de diez (10) estudiantes de sexto (6) de secundaria de la Institución Educativa Departamental rural de Palmira del municipio de Pueblo Viejo del Departamento del Magdalena. Cabe destacar que los participantes diligenciaron debidamente el consentimiento informado.

\section{Técnicas e instrumentos}

Se utilizaron en la recolección de información técnicas como la observación participante, grupos de discusión ayudando al intercambio de ideas en pro revisar qué factores influyen en las tecnologías de la información y comunicación en el desarrollo investigativo escolar y diarios de campo donde se consignaron todas las experiencias pedagógicas, observadas en el proceso de investigación.

\section{Procedimiento}

Este estudio utilizo la observación participante, grupos de discusión y diarios de campo procediendo a sistematizar la información recogida en el proceso de investigación (Ver tabla 1). 
TABLA 1.

Sistematización de las actividades apoyadas en TIC para el proceso investigativo

\begin{tabular}{ll}
\hline \multicolumn{1}{c}{ Fases } & \multicolumn{1}{c}{ Estrategia } \\
\hline Fase diagnóstica & $\begin{array}{l}\text { Los estudiantes seleccionaron una temática que fuera de su } \\
\text { interés, iniciaron viendo videos y revisando diferentes juegos y } \\
\text { aplicaciones donde se pudiera trabajar el tema. } \\
\text { Fase de desarrollo } \\
\text { Se seleccionaron las aplicaciones educativas pertinentes para } \\
\text { tema los temas seleccionados. }\end{array}$ \\
implementación & $\begin{array}{l}\text { Los estudiantes desarrollaron los contenidos programáticos } \\
\text { mediante el uso de plataformas virtuales, aplicaciones } \\
\text { educativas y juegos interactivos entre docentes y estudiantes. }\end{array}$ \\
& $\begin{array}{l}\text { Los docentes realizaron el proceso de evaluación donde midieron } \\
\text { los conocimientos que se debían adquirir luego del proceso de } \\
\text { Fase de evaluación }\end{array}$ \\
\hline
\end{tabular}

Fuente: elaboración propia, (2018)

\section{Resultados}

A continuación, se presentan los principales hallazgos del estudio, los cuales se basaron en la investigación acción participación en la implementación y resultados del estudio. Luego de la implementación de las estrategias que influyeron en las tecnologías de la información y comunicación en el desarrollo investigativo escolar se observó que los estudiantes tienen mayor disposición y se encuentran más receptivos a la información, mediante el uso de las nuevas tecnologías logrando realizar proceso de indagación, revisión bibliográfica, de contenidos digitales, mediante grupos de aprendizaje colaborativo, realizaron un dialogo de saberes donde intercambiaron conocimientos adquiridos sobre el uso de diversas aplicaciones en el ámbito educativo para el desarrollo de diversas asignaturas, encontrando estrategias pedagógicas basadas en la tecnología diseñadas de forma transversal, puesto que son técnicas que se pueden utilizar para la enseñanza de cualquier área del conocimiento.
Hoy en día se evidencia una gran atracción por la tecnología, los jóvenes y niños desarrollan cierto interés por el uso de herramientas tecnológicas para la realización de diversas actividades, el hablar con algún amigo, el realizar sus compromisos, el estudiar y documentarse, el mantenerse informado sobre lo que ocurre en el mundo, influyendo de manera positiva al momento de implementarlas en el aula de clase, por esto el uso de las tecnologías en las escuelas resulta ser de gran importancia ya que aporta más capacidad de información en la que los estudiantes podrían apoyarse para adquirir conocimiento.

Los maestros identifican que la tecnología es una herramienta de mucha importancia para facilitar los procesos de enseñanza-aprendizaje, pero para los chicos parece no sentirlo así, por cuanto ven en ella, un mecanismo de entretenimiento que los atrae más que la búsqueda del saber o la ayuda para realizar sus trabajos e investigaciones, por eso la idea de implementar la tecnología como herramienta durante el desarrollo de las clases con la finalidad de 
conseguir que los estudiantes le den un mayor aprovechamiento.

Como resultado de esta aplicación se espera que los estudiantes usen la tecnología como herramienta de aprendizaje más que como método de entretenimiento y esto a su vez ayude a subir el rendimiento de los estudiantes, debido a la facilidad de acceso de información que brinda el internet.

\section{Discusión}

A partir de lo estudiado en el trayecto de la investigación y recolección de información en la cual arrojaron resultados sustentados de lo planteado Hermosa, (2014) quien manifiesta que la utilización constante de los diferentes medios tecnológicos aplicándolos en el contexto educativo ha presentado un cambio que impacta en las nuevas exigencias, en el contexto de las instituciones puesto que trabajan con el compromiso de contribuir en el aprendizaje-practico.

Donde se asocia a lo que manifiesta Santacoloma, (2014). cuando dice que fomentar la investigación desde los diferentes escenarios educativos, busca lograr que asesores, maestros (as), niños,(as) y jóvenes, establezcan un dialogo de saberes mediado por una formación en investigación, que integrada al aula de clase permita cultivar el espíritu científico en los estudiantes desde edades tempranas, creando una cultura de investigación, que a su vez posibilite una educación basada en ciencia tecnología e innovación, convirtiéndose en un aporte para el desarrollo del país.

De esta manera el uso de la tecnología como herramienta de trabajo en las escuelas, ayuda a aumentar el rendimiento académico de los estudiantes, debido a la amplia gama de información que esta nos brinda, no importa que tan lejos se encuentre el alumno del docente a través de computadores se podrán comunicar y compartir información, para la elaboración de trabajos en casa es ideal pues permite acceder a información necesaria para su elaboración.

Hay que darle un correcto uso a esta ventaja que hoy en día tenemos, más que pensar en entretenimientos, pensemos en que podemos cambiar el mundo usando la información que en ella podemos encontrar. Lo cual se apoya en la visión de Graells, (2012) quien expresa que, las Tecnologías de la Información y las Comunicación (TIC) forman parte de la cultura tecnológica. Estas permiten ampliar las capacidades físicas y mentales de los individuos. Generando diversas posibilidades de desarrollo social y educativo. Las TIC no solo se encuentran asociadas a la informática o tecnología, sino que también incluye los medios de comunicación. En ese sentido, las TIC se convierten en un instrumento cada vez más indispensable en las instituciones educativas donde pueden realizar múltiples funcionalidades.

\section{Referencias}

Angustias, M., y Fernández, A. (2012). El aprendizaje semipresencial o virtual: nueva metodología de aprendizaje en Educación Superior. Revista Latinoamericana de Ciencias Sociales, Niñez y Juventud, 10(1).

Aretio, L. (2001). La educación a distancia. De la Teoría a la Práctica. Barcelona, Editorial Ariel.

Avendaño, I., Cortés, O., y Guerrero, H. (2015). Competencias sociales y tecnologías de la información y la comunicación como factores asociados al desempeño en estudiantes de básica primaria con experiencia de desplazamiento forzado. Diversitas, 11(1), 13-36.

Belloch (2012). Las Tecnologías de la Información y Comunicación en el aprendizaje. Universidad de Valencia. 
Bonilla, Castro, Elsy, 1942.Más allá del Dilema de los Métodos: La investigación en Ciencias Sociales. Bogotá: Grupo Editorial Norma, 2005. 424 p.

Camargo, C. (2015). La investigación como estrategia pedagógica en la Guajira, desde una perspectiva de la inclusividad y diversidad como nuevo paradigma para el desarrollo de habilidades, destrezas y competencias. Educación y ciudad, (29), 149-162.

Cárdenas, I., Zermeño, M., y Tijerina, R. (2013). Tecnologías educativas y estrategias didácticas: criterios de selección. Revista educación y tecnología, (3), 190206.

Delgado, M. (2014). La educación básica y media en Colombia: retos en equidad $y$ calidad.

Gil, A., Feliu, J., Rivero, I., y Gil, E. (2003). ¿Nuevas tecnologías de la información y la comunicación o nuevas tecnologías de relación? Niños, jóvenes y cultura digital. Consultado en octubre, 12, 2007.

Graells, P. (2013). Impacto de las TIC en la educación: funciones y limitaciones. Cuadernos de desarrollo aplicados a las TIC, 2(1), 2.

Guerra, C. (2013). La influencia de las nuevas tecnologías en el proceso de enseñanza aprendizaje de los estudiantes del Séptimo año de Educación Básica del paralelo " $A$ " $y$ " $B$ " de la escuela "Dr. Elías Toro Funes" de la parroquia de Quisapincha del cantón Ambato (Bachelor's thesis).

Hermosa Del vasto, Paola., M. (2015). Influencia de las tecnologías de información y comunicación (TIC) en el proceso enseñanza-aprendizaje: una mejora de las competencias digitales. Rev. Cient. Gen. José María Córdova 13(16), 121132
Herrera L, (2012). El rol del docente ante la aplicación de tecnologías para los procesos formativos.

Hernández, R., Fernández, C., y Baptista, P. (2010). Metodología de la investigación. Editorial Mc Graw Hill. México.

Lozano, M. (2011). Utilización de las nuevas tecnologías en el logro de aprendizajes significativos en la asignatura de biología en los estudiantes del bachillerato de la especialidad de química y biología del Colegio Experimental Bernardo Valdivieso de la ciudad de Loja. Periodo lectivo 2009-2010. Lineamientos alternativos (Bachelor's thesis).

Mejía, M., y Manjarrés, M. (2010). Las pedagogías fundadas en la investigación. Búsquedas en la reconfiguración de la educación. Revista Internacional Magisterio 42: 16-26. Colombia.

Ortega, P., Peñuela, D y López, D. (2009). Sujetos y prácticas de la pedagogía crítica. Ediciones El Búho. Bogotá.

Preciado, D. L. (2017). Análisis De Las Tic Como Herramientas De Comunicación Organizacional Para Potenciar La Participación En Clases De Los Estudiantes De Primero De Bachillerato En Administración De Sistema Informático Del Colegio Quevedo, 2017 (Doctoral Dissertation, Universidad De Guayaquil).

Riveros, V., y Mendoza, M. (2005). Bases teóricas para el uso de las TIC en Educación. Encuentro educacional, 12(3).

Said, E., Irirarte, F., Valencia, J., Borjas, M., Ordoñez, M., Arellano, W., Román, G., Mestre, G., Payares, M., Lara, J., Jabba, D., Brändie, G., González, E. y Mejía, L. (2015). Hacia el fomento de las TIC en el sector educativo en Colombia. Ed:Uninorte. Colombia. 
Santacoloma, A. (2012). Los semilleros de investigación como estrategia de formación integral para ciudadanos del tercer milenio. Cultura Educación y Sociedad 3 (1): 13-21.

Salas, R. (2002). La calidad en el desarrollo profesional: avances y desafíos. Educación Médica Superior, 14(2), 136-147.

Silva, I., Borrero, A. M., Marchant, P., González, G., y Novoa, D. (2006). Percepciones de jóvenes acerca del uso de las tecnologías de información en el ámbito escolar. Última década, 14(24), 37-60.
Vargas, R. (2007). Exclusión, marginación y desarrollo de los pueblos indígenas. Ra Ximhai: revista científica de sociedad, cultura y desarrollo sostenible, 3(3), 693-706.

Waldegg, G. (2002). El uso de las nuevas tecnologías para la enseñanza y el aprendizaje de las ciencias. Revista electrónica de investigación educativa, 4(1), 01-22. 